\title{
ASPECTOS DA DISTRIBUIÇÃO DE PANTOPODA EM SARGASSUM DE ITANHAÉM E UBATUBA, SÃO PAULO, BRASIL
}

\author{
Franci Mary Fantinato Varoli ${ }^{1}$
}

\begin{abstract}
ASPECTS OF DISTRIBUTION OF PANTOPODA ON SARGASSUM FROM ITANHAÉM and UbatubA, Sĩo PAulo, Brazil. Qualitative and quantitative aspects of the distribution of Pantopoda species living on Sargassum of Ubatuba and Itanhaém (northern and southern coast at São Paulo State, respectively) were studied. The results showed the occurrence of nine Pantopoda species. The highest relative frequency was assigned to Tanystylum isabellae Marcus, 1940. In general the density of Pantopoda species was related to that of Hydrozoa covering Sargassum.

KEY WORDS. Pantopoda, Sargassum fauna, distribution, São Paulo
\end{abstract}

Os pantópodes embora não sejam organismos dominantes na fauna bentônica, podem ser considerados ubíquos. Dentre os trabalhos, mais recentes, realizados com esses organismos em diferentes partes do mundo, há os de DE HARO (1978) que comparou espécies de pantópodes presentes em algas e fanerógamas, o de RIBEIRO et al. (1982) que descreveram espécies encontradas em pequena profundidade, os de CHILD $(1982)$ e STOCK $(1986,1987)$ que realizaram estudos com espécies abissais, de CHIMENZ (1986) que estudou pantópodes de fundos inconsolidados e de STOCK (1992) que descreveu espécies do sul do Brasil.

O objetivo do presente estudo foi determinar, quali-quantitativamente, as espécies de pantópodes que vivem em Sargassum C. Agardh, 1820 em praias de intensidades de ondas diferentes.

\section{MATERIAL E MÉTODOS}

O material analisado foi coletado em quatro costões no estado de São Paulo, situados em Ubatuba (23⒊ $30^{\prime} \mathrm{S}$ e $45^{\circ} 06^{\prime} \mathrm{W}$ ) - Praia Grande e Praia do Lamberto (Fig. 1), consideradas respectivamente com alta e baixa energia e em Itanhaém $\left(24^{\circ} 12^{\prime} \mathrm{S}\right.$ e $46^{\circ} 47^{\prime} \mathrm{W}$ ) - Prainha (alta energia) e Praia de Peruíbe (baixa energia) (Fig. 2).

As praias estudadas eram ricas em Sargassum o qual ocorrem na franja do infralitoral. Duas espécies foram identificadas nos locais de coleta: Sargassum cymosum C. Agardh, 1820 e Sargassum rigidulum Kuetzing, 1845. Na Praia Grande, Praia do Lamberto e Praia de Peruíbe foi coletado $S$. cymosum sendo que as plantas das praias consideradas expostas (alta energia) apresentavam menor tamanho em comparação àquelas de praias consideradas abrigadas (baixa energia). $\mathrm{Na}$ Prainha a espécie coletada foi S. rigidulum.

1) Instituto Oceanográfico, Universidade de São Paulo. Praça do Oceanográfico 191, 05508-900 São Paulo, São Paulo, Brasil. Pesquisadora do CNPq. 


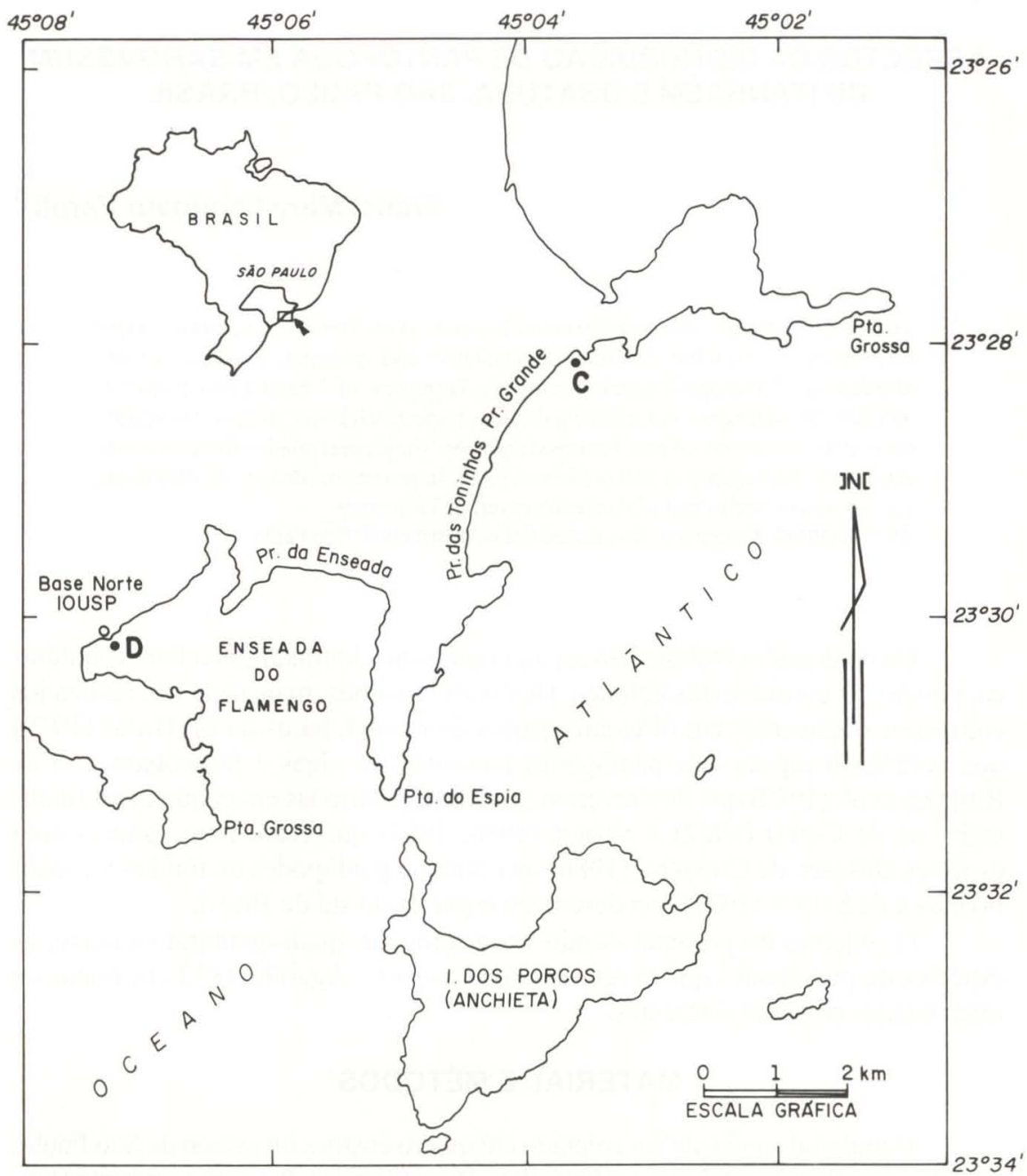

Fig. 1. Mapa da região de Ubatuba, indicando os locais de coleta. (C) Praia Grande, (D) Praia do Lamberto.

As coletas foram realizadas mensalmente em maré baixa. Em Ubatuba durante junho/1974 a maio/1975 e em Itanhaém de abril/1977 a março/1978.

As algas foram retiradas do substrato, fixadas no campo em formol a $4 \% \mathrm{e}$ transferidas posteriormente para álcool $70 \%$. Os pantópodes encontrados foram separados por espécie e calculada a freqüência relativa mensal.

Para as amostras de Itanhaém, após a retirada dos animais, mediu-se o fitovolume pelo método de deslocamento de líquidos, segundo DOMMASNES (1969) e calculou-se a densidade mensal (número de indivíduos por unidade de volume) para o número total de pantópodes. 


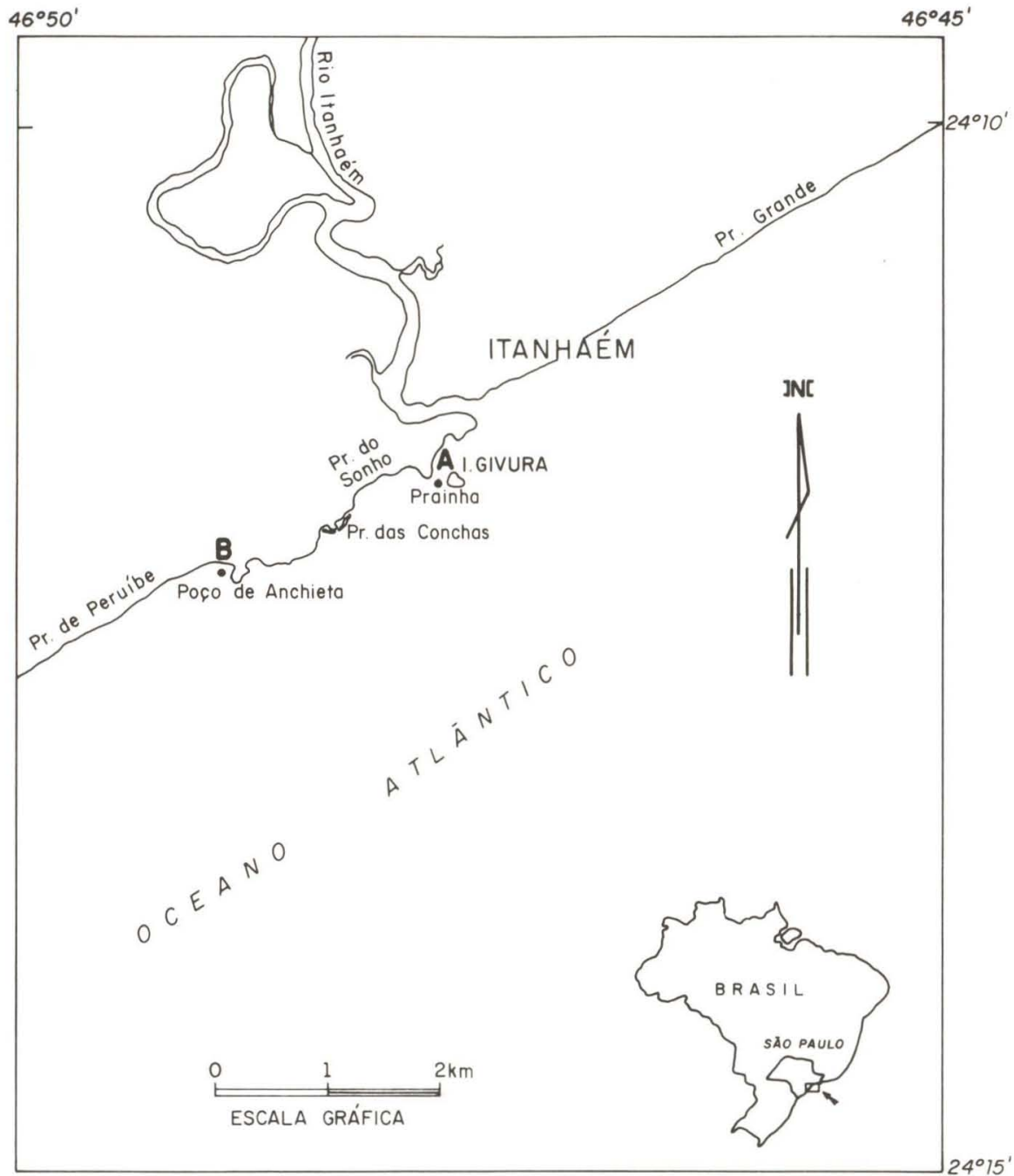

Fig. 2. Mapa da região de Itanhaém, indicando os locais de coleta. (A) Prainha, (B) Praia de Peruibe.

A densidade de pantópodes encontrada mensalmente em Itanhaém, foi comparada com a presença de hidrozoários sobre as algas. Para a avaliação relativa da quantidade dos mesmos, estipulou-se um critério arbitrário, levando-se em conta sua distribuição na superfície das algas. Assim, quando a existência de hidrozoários era de $75 \%$ a $100 \%$ de recobrimento, atribuiu-se o valor $\mathrm{A}$, de $50 \%$ a $75 \%$ o valor $\mathrm{A}^{\prime}$ e de $25 \%$ a $50 \% \mathrm{~A}^{\prime \prime}$, menor que $25 \%$ o valor A','. 
Tabela I. Freqüência ralativa mensal das espécies de Pantopoda de Itanhaém. (P) Prainha, (P.P.) Praia de Peruibe.

\begin{tabular}{|c|c|c|c|c|c|c|c|c|c|c|c|c|}
\hline \multirow{2}{*}{ Espécies } & \multicolumn{2}{|c|}{ Abril } & \multicolumn{2}{|c|}{ Maio } & \multicolumn{2}{|c|}{ Junho } & \multicolumn{2}{|c|}{ Julho } & \multicolumn{2}{|c|}{ Agosto } & \multicolumn{2}{|c|}{ Setembro } \\
\hline & $\mathrm{P}$ & P.P. & $\mathrm{P}$ & P.P. & $P$ & P.P. & $P$ & P.P. & $P$ & P.P. & $P$ & P.P. \\
\hline A. sawayai & - & - & - & 4,76 & - & - & - & - & - & - & - & - \\
\hline A. appendiculata & 76,19 & 66,66 & 36,84 & 14,28 & 24,00 & 10,52 & 5,49 & 12,50 & - & 7,14 & 5,12 & 5,55 \\
\hline C. evelinae & - & 19,04 & - & 5,26 & - & 50,00 & - & 14,28 & - & 22,22 & - & 10,71 \\
\hline A. evelinae & 9,52 & - & 1,97 & - & 4,00 & - & - & - & 2,56 & - & - & - \\
\hline A. stictus & - & - & 2,63 & 47,61 & - & 26,31 & 9,89 & 18,75 & 5,12 & 57.14 & 17,94 & 27,77 \\
\hline P. pamphorum & - & 33,33 & - & 4,76 & 2,00 & 10,52 & - & - & - & - & - & - \\
\hline T. isabellae & 14,28 & - & 58,55 & 9,52 & 70,00 & 47,36 & 84,61 & 18,75 & 92,30 & 21,42 & 76,92 & $4 \check{4}, 44$ \\
\hline
\end{tabular}

(Continua)

Tabela I. Continuação.

\begin{tabular}{|c|c|c|c|c|c|c|c|c|c|c|c|c|}
\hline \multirow{2}{*}{ Espécies } & \multicolumn{2}{|c|}{ Outubro } & \multicolumn{2}{|c|}{ Novembro } & \multicolumn{2}{|c|}{ Dezembro } & \multicolumn{2}{|c|}{ Janeiro } & \multicolumn{2}{|c|}{ Fevereiro } & \multicolumn{2}{|c|}{ Março } \\
\hline & $P$ & P.P. & $P$ & P.P. & $P$ & P.P. & $P$ & P.P. & $P$ & P.P. & $P$ & P.P. \\
\hline A. sawayai & - & 3,57 & - & - & - & - & - & 33,33 & - & - & - & - \\
\hline A. appendiculata & 10,52 & 10,71 & - & 25,00 & 33,33 & 13,33 & - & $-\quad:$ & 14,28 & 28,57 & - & 75,00 \\
\hline C. evelinae & 18,42 & - & 16,16 & - & - & - & - & - & - & - & - & - \\
\hline A. evelinae & 2,63 & 3,57 & 33,33 & 25,00 & - & - & 33,33 & - & 28,57 & - & 33,33 & - \\
\hline A. stictus & 2,63 & 35,71 & 16,66 & - & - & 60,00 & 66,66 & 33,33 & - & 42,85 & 33,33 & 25,00 \\
\hline P. pamphorum & 5,26 & - & - & - & 33,33 & 6,66 & - & 33,33 & 57,14 & - & - & - \\
\hline T. isabellae & 60,52 & 35,71 & 33,33 & 50,00 & 33,33 & 20,00 & - & - & - & 28,57 & 33,33 & - \\
\hline
\end{tabular}

\section{RESULTADOS}

As espécies obtidas foram: Achelia sawayai Marcus, 1940; Ammothella appendindiculata (Dohrn, 1881); Nimphopsis melidae Sawaya, 1947; Endeis spinosa Montagu, 1808; Callipallene evelinae Marcus, 1940; Anoplodactylus evelinae Marcus, 1940; Anoplodactylus stictus Marcus, 1940; Pycnogonum stictus Marcus, 1940 e Tanystylum isabellae Marcus, 1940. As espécies que ocorreram nas quatro praias foram: A. appendiculata, A. stictus, A. evelinae e T. isabellae . C. evelinae foi coletada nas duas praias de Itanhaém e somente na praia exposta de Ubatuba. $A$. sawayai foi obtida apenas em praias abrigadas. N. melidae e E. spinosa ocorreram respectivamente nas praias abrigadas e exposta de Ubatuba, não sendo registrada suas presenças em Itanhaém. Foram obtidos 949 indivíduos nos quatro locais de coleta. Em Itanhaém foram coletados 616 exemplares, sendo 462 na Prainha e 154 na Praia de Peruíbe. Em Ubatuba foram obtidos 333 indivíduos, dos quais 129 foram coletados na Praia Grande e 204 na Praia do Lamberto.

Na Prainha e Praia de Peruíbe (Tab. I) as espécies A. appendiculata, T. isabellae e $A$. stictus foram as mais ocorrentes. As demais espécies não ocorreram em todos os meses e quando presentes, suas freqüências relativas foram notadamente menores.

Na Praia Grande e Praia do Lamberto (Tab. II) A. stictus e T. isabellae foram as espécies mais freqüentes e com maiores valores de freqüência relativa. As outras espécies ocorreram esporadicamente e com freqüências inferiores . 
Tabela II. Freqüência relativa mensal das espécies de Pantopoda de Ubatuba. (P.G.) Praia Grande. (P.L.) Praia do Lamberto.

\begin{tabular}{|c|c|c|c|c|c|c|c|c|c|c|c|c|}
\hline \multirow{2}{*}{ Espécies } & \multicolumn{2}{|c|}{ Julho } & \multicolumn{2}{|c|}{ Agosto } & \multicolumn{2}{|c|}{ Setembro } & \multicolumn{2}{|c|}{ Outubro } & \multicolumn{2}{|c|}{ Novembro } & \multicolumn{2}{|c|}{ Dezembro } \\
\hline & P.G. & P.L. & P.G. & P.L. & P.G. & P.L. & P.G & P.L. & P.G. & P.L. & P.G. & P.L. \\
\hline A. appendiculata & - & 12,50 & - & 9,67 & - & 14,28 & - & 4,00 & - & 13,88 & - & 15,78 \\
\hline A. evelinae & 3,70 & - & - & 3,22 & - & 14,28 & - & - & - & - & - & - \\
\hline A. sawayai & - & 19,35 & - & - & - & - & - & - & - & - & - & - \\
\hline A. stictus & 29,62 & - & 42,85 & 16,12 & 45,16 & 14,28 & 33,33 & 20,00 & 22,22 & 25,00 & - & 47,36 \\
\hline C. evelinae & - & - & - & - & - & 14,28 & - & 8,00 & - & - & - & 10,52 \\
\hline E. spinosa & - & - & - & - & - & - & - & - & - & - & - & - \\
\hline N. melidae & - & - & - & - & - & - & - & - & - & - & - & 5,26 \\
\hline P. pamphorum & - & - & - & - & - & - & 6,66 & - & 5,55 & - & - & - \\
\hline T. isabellae & 66,66 & 87,50 & 57,14 & 51,61 & 54,83 & 42,85 & 60,00 & 68,00 & 72,22 & 61,11 & - & 21,05 \\
\hline
\end{tabular}

(Continua)

Tabela II. Continuacão.

\begin{tabular}{|c|c|c|c|c|c|c|c|c|c|c|c|c|}
\hline \multirow{2}{*}{ Espécies } & \multicolumn{2}{|c|}{ Janeiro } & \multicolumn{2}{|c|}{ Fevereiro } & \multicolumn{2}{|c|}{ Março } & \multicolumn{2}{|c|}{ Abril } & \multicolumn{2}{|c|}{ Maio } & \multicolumn{2}{|c|}{ Junho } \\
\hline & P.G. & P.L. & P.G. & P.L. & P.G. & P.L. & P.G & P.L. & P.G. & P.L. & P.G. & P.L. \\
\hline A. appendiculata & 6,25 & 12,50 & - & - & - & - & - & - & - & - & - & - \\
\hline A. evelinae & - & - & - & - & - & - & - & - & - & - & - & - \\
\hline A. sawayai & - & - & - & - & - & - & - & - & - & - & - & - \\
\hline A. stictus & 12,25 & 12,50 & - & - & 50,00 & - & 80,00 & 23,80 & 25,00 & - & - & 5,00 \\
\hline C. evelinae & - & - & - & 20,00 & - & - & - & - & - & - & - & - \\
\hline E. spinosa & - & - & - & - & - & - & - & - & 25,00 & - & - & - \\
\hline N. melidae & - & - & - & - & - & - & - & - & - & - & - & 5,00 \\
\hline P. pamphorum & - & - & - & - & - & - & - & - & - & - & - & - \\
\hline T. isabellae & 81,25 & 75,00 & - & 80,00 & 50,00 & 100,00 & 20,00 & 76,19 & 50,00 & 100,00 & 100,00 & 90,00 \\
\hline
\end{tabular}

A densidade relativa calculada para o número total de indivíduos obtidos em Itanhaém foi maior na Prainha, durante todo o período de coleta, com exceção do mês de dezembro quando foi maior na Praia de Peruíbe. Em fevereiro as duas praias apresentaram a mesma densidade (Tab. III). Ressalta-se que os meses de abril a setembro, na Prainha, foram os meses em que o hidrozoário Sertularia sp. foi mais abundante e foram também os meses de maior densidade de pantópodes (Tab. III).

O Sargassum da Praia de Peruíbe apresentou durante o período de coleta um menor recobrimento de hidrozoário do que a Prainha, assim como a menor densidade de pantópodes (Tab. III).

\section{DISCUSSÃO E CONCLUSÕES}

Considerando-se que a maioria dos trabalhos brasileiros sobre pantópodes referem-se a Sistemática, sendo poucos os estudos ecológicos existentes e em termos de dados quantitativos os autores têm utilizado diferentes metodologias, os resultados aqui obtidos não puderam ser devidamente comparados com a literatura disponível.

O número de indivíduos obtidos foi relativamente pequeno comparando-se com outros grupos que ocorrem no fital conforme também constatado por MUKAI (1971), TARARAM \& WAKABARA (1981) e MASUNARI (1982). 
Tabeal III. Densidade de Pantopoda e o recobrimento de hidrozoários sobre Sargassum em itanhaém. $A=75-100 \%$. $A^{\prime}=50-75 \%$. $A^{\prime \prime}=25-50 \%$. $A^{\prime \prime \prime}=$ menor aue $25 \%$.

\begin{tabular}{|c|c|c|c|c|}
\hline \multirow{2}{*}{ Meses } & \multicolumn{2}{|l|}{ Prainha } & \multicolumn{2}{|c|}{ Praia de Peruibe } \\
\hline & Densidade Pantopoda & Hidrozoa & Densidade Pantopoda & Hidrozoa \\
\hline Abril/77 & 0,083 & $A$ & 0,008 & $A^{\prime \prime \prime}$ \\
\hline Maio/77 & 1,006 & A & 0,044 & $A^{\prime \prime \prime}$ \\
\hline Junho/77 & 0,277 & A & 0,066 & $A^{\prime \prime \prime}$ \\
\hline Julho/77 & 0,543 & $A^{\prime \prime}$ & 0,090 & $A^{\prime \prime \prime}$ \\
\hline Agosto/77 & 0,160 & $A^{\prime \prime \prime}$ & 0,060 & $A^{\prime \prime \prime}$ \\
\hline Setembro/77 & 0,155 & $A^{\prime \prime}$ & 0,050 & $A^{\prime \prime \prime}$ \\
\hline Outubro/77 & 0,110 & $A^{\prime \prime \prime}$ & 0,067 & $A^{\prime \prime \prime}$ \\
\hline Novembro/77 & 0,020 & $A^{\prime \prime \prime}$ & 0,010 & $A^{\prime \prime \prime}$ \\
\hline Dezembro/77 & 0,013 & $A^{\prime \prime \prime}$ & 0,050 & $A^{\prime \prime \prime}$ \\
\hline Janeiro/78 & 0,047 & $A^{\prime \prime \prime}$ & 0,036 & $A^{\prime \prime \prime}$ \\
\hline Fevereiro/78 & 0,020 & $A^{\prime \prime \prime}$ & 0,020 & $A^{\prime \prime \prime}$ \\
\hline Março/78 & 0,015 & $A^{\prime \prime \prime}$ & 0,010 & $A^{\prime \prime}$ \\
\hline
\end{tabular}

A análise das freqüências relativas confirmam o domínio de Tanystylum isabellae seguido por Anoplodactylus stictus nas praias estudadas. Esses dados concordam com MASUNARI (1982), que considera T. isabellae uma espécie presente no fital de Amphiroa beauvoisii e A. stictus com ocorrências variáveis ao longo do ano.

Os pantópodes ingerem diferentes tipos de alimentos (VAROLI 1994) porém, um dos fatores fundamentais na ocorrência desses organismos é a presença de hidrozoários considerados como a principal fonte alimentar (MARCUS 1940; FRY 1965; KING 1973). Dessa forma, sugere-se que a variação nas densidades dos pantópodes nas praias estudadas em Itanhaém esteja relacionada diretamente a presença de Sertularia sp..

AGRADECIMENTOS. Agradeço a Dra Patricia Teresa Monteiro Cunningham pela revisão do texto.

\section{REFERÊNCIAS BIBLIOGRÁFICAS}

CHILD, C.A. 1982. Deep sea Pycnogonida from the North and South Atlantic Basins. Smiths Contr. Zool. 349: 1-52.

ChimenZ, C. 1986. Soft bottom Pycnogonida from the Gulf of Salermo (Italy) Oebalia 12: 37-146.

DE HARO, A. 1978. Ecological distribution of pycnogonids on the Catalan Coast. Zool. Jour. Limn. Soc. 63 (1/2): 181-196.

DOMMASNES, A. 1969. On the fauna of Corallina officinalis L. in the western Norway. Sarsia 38: 71-86.

FRY, W.G. 1965. The feeding mechanisms and preferred foods of three species of Pyenogonida. Bull. Brit. Mus. (Nat. Hist), Zool. 12 (6): 197-223. 
KING, P.E. 1973. Pycnogonids. London, Hutchinson, 144p.

MARCUS, E. 1940. Os Pantopoda brasileiros e os demais sulamericanos. Bolm Fac.

Filos. Ciênc. Univ. S. Paulo 4: 2-144.

MAsUnARI, S. 1982. Organismos do Fital de Amphiroa beauvoisii Lamourox, 1816 (Rodophyta: Corallinaceae), I. Auto-ecologia. Bolm Inst. oceanogr. 12 (3): 1-8. MuKaI, H. 1971. The phytal animals on the thalli of Sargassum serratifolium in the Sargassum region with reference to their seasonal fluctuations. Mar. Biol. 8(2): 170-182.

Ribeiro, M.A.G.; P. ACÂNTARA Filho; F.M. FAnTinato \& L.R. TOMMASI. 1982.

Sobre a ocorrência de Pantopoda na Região da Baía de Santos, São Paulo (Brasil). Dusenia, Curitiba, 13 (1): 37-40.

Stock, J.H. 1986. Pycnogonida from the Caribbean and Straits of Florida. Bull. mar. Sci. 38 (3): 399-441. 1987. Faunistics transit between the Atlantic and the Mediterranean: The deep-water Pycnogonida. Cah. Bio. mar. 28: 505-519.

. 1992. Pycnogonida from Southern Brazil. Tijd. Ent. 135: 113-139.

TARARAM, A.S. \& Y. WAKABARA. 1981. The mobile fauna-especially Gammaridea of Sargassum cymosum. Mar. Ecol. Prog. Ser. 5 (2): 157-163.

VAROLI, F.M.F. 1994. Aspectos da alimentação de Tanystylum isabellae e Anoplodactylus stictus (Pantopoda). Revta bras. Zool. 11 (4): 623-627.

Recebido em 04.X.1994; aceito em 13.V.1996. 